\title{
Studies on gastric mucosal microcirculation. 1. The nature of regional variations induced by ethanol injury
}

\author{
N Kalia, N J Brown, S Jacob, M W R Reed, K D Bardhan
}

\begin{abstract}
Background/Aims-The focal nature of gastric ulcers raises the possibility of underlying regional disturbances in gastric mucosal microcirculation. This study employed fluorescent in vivo microscopy with the aim of directly investigating the response of several areas of the gastric mucosa to $60 \%$ ethanol.

Methods-Changes in macromolecular leakage of fluorescein labelled albumin, vessel diameter, and acridine red labelled leucocyte adhesion and rolling were assessed over a period of two hours. A total of $0.5 \mathrm{ml} 60 \%$ ethanol was topically applied for five minutes to the exteriorised gastric mucosa of anaesthetised rats.

Rats-Three distinct patterns of response were found. Areas of lesion formation were small and occurred within five minutes. These areas showed persistent blood flow stasis throughout the course of the experiment, increased leakage $(p<0.02)$, and no leucocyte adhesion. Peripheral to the lesion, sustained leakage $(\mathbf{p}<0.02)$ was found with adherence of leucocytes $(p<0.01)$ after lesion formation. Sites more remote to any lesion showed transient leakage and significant numbers of 'rolling' leucocytes $(p<0 \cdot 01)$ were observed again after the lesion had formed.

Conclusions-Despite widespread exposure of the entire gastric mucosa to $60 \%$ ethanol the resultant mucosal injury was limited. Widespread vascular damage was found reflected by macromolecular leakage, the pattern of which showed regional variation.

(Gut 1997; 40: 31-35)
\end{abstract}

Biomedical Science, University of Sheffield, Sheffield

N Kalia

S Jacob

\section{Department of} Surgical and Anaesthetic Sciences, Royal Hallamshire Hospital, Sheffield N J Brown M W R Reed

\section{Rotheram District} General Hospital NHS Trust, Rotherham K D Bardhan

Correspondence to: Neena Kalia, Department of Biomedical Ucience, Western Bank, Sheffield, S10 2TN.

Accepted for publication 29 July 1996
Keywords: ethanol injury, gastric microcirculation, in vivo microscopy, leucocytes, macromolecular leakage, endothelial adhesion.

The traditional concept of ulcer formation is an imbalance between the 'attack' factors (acid, pepsin) and the 'defence' factors (HCO3-, prostaglandin generation). However, if this were the sole explanation then mucosal damage would be likely to be more widespread, whereas peptic ulcers are areas of focal necrosis. This in turn raises the possibility of 'regional' microcirculatory disturbances leading to focal ischaemia, weakening, and then to ulceration.

The evidence for a potential role for the gastric mucosal microcirculation in ulcer formation is complicated by lack of direct evidence linking impaired blood flow with ulceration. The finding of much reduced tissue type plasminogen activator at the ulcer edge ${ }^{1}$ provides support for this as do the few experimental studies that have used histology to show blood flow changes occurring before the development of gastric ulcers. ${ }^{2-4}$ There is also evidence that links the distribution and focality of intestinal ulcers with the microvascular anatomy. ${ }^{5}$ This has been reviewed by Piasecki, ${ }^{6}$ who suggests that the presence of end arteries may make tissue more susceptible to damage.

Most studies have concentrated on the microcirculatory events occurring at the site of lesion formation. We have used fluorescent in vivo microscopy to directly examine the response of several areas of the mucosal microcirculation to ethanol, which allowed for areas that recovered from injury to also be assessed. Specifically, alterations in blood flow, macromolecular leakage, and vessel diameters were quantified. The role of leucocytes (adhesion and rolling) has received little attention in ethanol induced injury, so they were also studied.

\section{Methods}

ANIMALS

Experiments were carried out on 24 adult male Wistar rats weighing between 100 and $150 \mathrm{~g}$ obtained from the University of Sheffield Field Laboratories. Animals were fasted overnight before experimentation and housed individually in wire bottomed cages to prevent coprophagy. Experimental procedures were carried out in accordance with Home Office approval.

Animals were anaesthetised with a subcutaneous injection of diazepam $(5 \mathrm{mg} / \mathrm{ml}$, Dumex Ltd) and Hypnorm $(0.315 \mathrm{mg} / \mathrm{ml}$ fentanyl citrate and $10 \mathrm{mg} / \mathrm{ml}$ fluanisone, Janssen Pharmaceutical Ltd) in the ratio of $1: 1$ at a volume of $0.1 \mathrm{ml} / 100 \mathrm{~g}$ body weight with more anaesthetic given subcutaneously as required.

\section{SURGICAL PROCEDURE}

Tracheotomy was performed and an oesophageal thermistor probe was inserted and connected to a thermometer (Fluke, Washington, USA). A cannula was placed in the left carotid artery and was connected to a 
pressure transducer and physiograph (MicroMed Louisville, USA) to monitor mean arterial blood pressure and heart rate and also to provide access for administration of fluorochromes.

After laparotomy a $1.0 \mathrm{~cm}$ incision was made by electric microcautery in the middle of the anterior wall of the exteriorised stomach parallel to the 'limiting ridge' (which separates the squamous cell lined forestomach from the more distal glandular stomach). The observations were made on the glandular portion of the stomach. Care was taken to avoid burning through blood vessels.

The animal was placed on its left side on a heating pad on a special perspex stage to maintain body temperature around $35-37^{\circ} \mathrm{C}$. A glass microscope slide was mounted on perspex pegs and the stomach was gently extended on to it and held in place by a stay suture. The incision made in the anterior wall was opened using microclamps to allow visualisation of the posterior mucosal surface. The stomach was kept warm by covering with Saran wrap - an impermeable membrane that also prevented dehydration.

\section{FLUORESCENT IN VIVO MICROSCOPY}

The animal, warming pad, and perspex board were transferred to the stage of a Leitz fluorescent microscope equipped with a mercury arc lamp for epi-illumination fluorescent light microscopy. A filter cube interposed into the light path of the mercury arc lamp permitted green light $(530-560 \mathrm{~nm})$ and blue light (450-490 nm) to be selected for epi-illumination. Images of the preparation were monitored using a cooled coupled device camera (CCD, Hitachi) displayed on a high resolution monitor (Sony PVM-1443) and recorded on videotape (Sony SLV-373-UB) for later off line analysis. All instruments were calibrated before each experiment.

After the surgical procedure, 30 minutes were allowed for blood pressure, heart rate, and body temperature to stabilise. These variables were then monitored every 15 minutes for the remainder of the experiment. After the stabilisation period either acridine red, which fluoresces under green light, $(0.1 \mathrm{ml} / 100 \mathrm{~g}$ body weight) or fluoroscein isothiocyanate conjugated to bovine serum albumin, which fluoresces under blue light, (FITC-BSA; $0.2 \mathrm{ml} / 100 \mathrm{~g}$ body weight) was injected via the carotid cannula. Acridine red labels leucocytes and FITC-BSA allows blood flow to be visualised. Under circumstances resulting in increased microvascular permeability to macromolecules, FITC-BSA can be seen to leak from the vasculature, appearing as a flare in the interstitium. The interstitial fluorescent intensity is proportional to the degree of FITC-BSA leakage from the vessels. $^{7}$

EXPERIMENTAL PROTOCOL

The animals were divided into four groups of six; two control groups were treated with distilled water and two experimental groups were treated with $60 \%$ ethanol.

Preliminary experiments confirmed that $60 \%$ ethanol consistently produced damage, reflected by leakage of FITC-BSA, but the actual areas of mucosal necrosis were randomly scattered. The microscopy technique does not allow the entire mucosa to be kept under surveillance simultaneously; therefore three areas were preselected in each animal, well removed from each other for detailed and continuous observation. These preselected areas included the characteristic honeycomblike network of mucosal capillaries and at least one postcapillary venule. ${ }^{8}$ Capillaries had a typical diameter of $10-12 \mu \mathrm{m}$ and drained into the postcapillary venules of 20-25 $\mu \mathrm{m}$ diameter. The location of these three areas were marked on the impermeable membrane. In effect the areas under observation were randomly selected. Changes in mucosal blood flow, macromolecular leakage, and vessel diameter were assessed. For the acridine red experiments only two areas of interest were identified as it was difficult to assess three areas simultaneously.

Measurements were recorded from all selected regions five minutes after the administration of the fluorochromes. Then either $0.5 \mathrm{ml}$ distilled water or $0.5 \mathrm{ml} 60 \%$ ethanol was applied topically to the exposed mucosa. This volume was sufficient to ensure that the entire exposed region was submerged. Five minutes later, the exposed mucosa was cleared of the water or ethanol by gentle aspiration and washed with saline kept at $37^{\circ} \mathrm{C}$. Post-treatment recordings were made at five minutes and then every 15 minutes for the next two hours. To ensure good resolution, the mucus was absorbed every 30 minutes using Kleenex medical wipes.

\section{DATA COLLECTION AND QUANTIFICATION}

\section{FITC-BSA leakage}

Postcapillary venules (PCVs) and capillaries were studied. To measure FITC-BSA leakage from the vessels, an area of interest adjacent to three segments of the PCV and surrounding three separate capillaries was mapped out on the television screen. Computerised image analysis (Image Pro Plus, Media Cybernetics, USA) was used to measure interstitial fluorescence at each time point during the experiment.

The image analysis programme stored the recorded image as a palette of $\mathbf{2 5 5}$ grey levels. A pretreatment grey level was taken and used as the baseline value. After ethanol treatment, the grey level was again measured at each time point. Because an increase in fluorescence represented a proportional increase in leak, values for percentage increase in leak could be calculated.

Vessel diameters and blood flow

Diameters of the PCVs and capillaries in the preselected areas were also measured by 
computerised image analysis calibrated to produce values in microns. Mucosal vessel blood flow was assessed qualitatively.

Leucocyte adhesion and rolling

The sum of adherent and rolling leucocytes in both PCVs and capillaries within a single field of view was determined off line during playback of videotaped images. A leucocyte was considered adherent to the vessel endothelium if it remained stationary for $\geq 30$ seconds. Adherent and rolling leucocytes were expressed as the number per field of view observed on the monitor during a period of one minute at each time point.

Leucocytes adherent within the blood vessel were often difficult to distinguish from those that had emigrated into the interstitial space; therefore both types were counted as being adherent. Any leucocytes that were adherent at the onset of the experiment were subtracted from the total number that accumulated subsequently.

STATISTICAL ANALYSIS

Vessel diameters and macromolecular leakage before and after ethanol application or distilled water were tested for within group variations using the Wilcoxon test for non-parametric data. Vessel diameters and macromolecular leakage in the experimental and control groups were tested for between group variations using the Mann-Whitney U test for non-parametric data. Changes in leucocyte activity was tested for significance by one tailed Student's $t$ test. Results were considered significant at $\mathrm{p} \leq 0.05$.

\section{Results}

GENERAL FINDINGS

In the FITC-BSA experiments, three areas of the exposed stomach were assessed in each of the six animals. Three general patterns in terms of blood flow, macromolecular leakage, and leucocyte adherence were noted in these 18 areas in response to ethanol:

(1) Areas of persistent blood blow stasis associated with mucosal necrosis $(n=7)$

(2) Areas of increased macromolecular leakage and leucocyte adherence at the periphery of the blood flow stasis zones $(n=4)$

(3) Areas of transient macromolecular leakage and rolling leucocytes in remote areas $(n=7)$

Areas of mucosal necrosis (confirmed histologically) were small, up to $2 \mathrm{~mm}$ in diameter, and randomly distributed. No more than three or four such areas were observed per stomach. Distilled water had no general effect either macroscopically or microscopically.

\section{VESSEL DIAMETERS}

Both PCVs and capillaries, regardless of their position, showed no change in vessel diameters after treatment. Distilled water had no effect on vessel diameters in control animals.

\section{BLOOD FLOW}

Blood flow was normal and at a steady velocity but decreased immediately in all vessels after topical application of ethanol. The congestion of red blood cells led to stasis within all vessels. In the seven areas where stasis persisted throughout the two hour duration of the experiment, macroscopically visible lesions developed within 5-10 minutes on the surface of the mucosa. Haemorrhage of these necrotic lesions often occurred within 10 minutes.

The four areas observed at the periphery of the lesion and the seven areas more remote showed recovery from the stasis. Within two to three minutes sluggish resumption of blood flow was found in these areas with flow returning to pretreatment velocities by five to six minutes. Distilled water had no effect on blood flow in control animals.

\section{MACROMOLECULAR LEAKAGE}

Topical application of ethanol to the gastric mucosa induced an immediate increase in the macromolecular leakage of FITC-BSA from both PCVs and capillaries in all areas. However, subsequent events varied according to the area observed. In the seven areas of persistent blood flow stasis an initial sharp significant increase $(p<0.02)$ in macromolecular leakage into the interstitium was observed after five minutes (Fig 1). The interstitial fluorescence then declined reaching a plateau which was still significantly $(p<0.02)$ higher than pretreatment and control values.

In the four areas at the periphery of a lesion, a similar and significant $(p<0.02)$ increase in macromolecular leakage was seen after five minutes which increased further after 15 minutes and remained significantly $(p<0.02)$ raised for the duration of the experiment (Fig 1).

The macromolecular leakage after five minutes was also significant $(p<0.02)$ in the seven areas remote from stasis (Fig 1). Thereafter it declined but remained higher than pretreatment values $(\mathrm{p}<0.05)$.

The graphical data illustrates the regional variations in macromolecular leakage in the

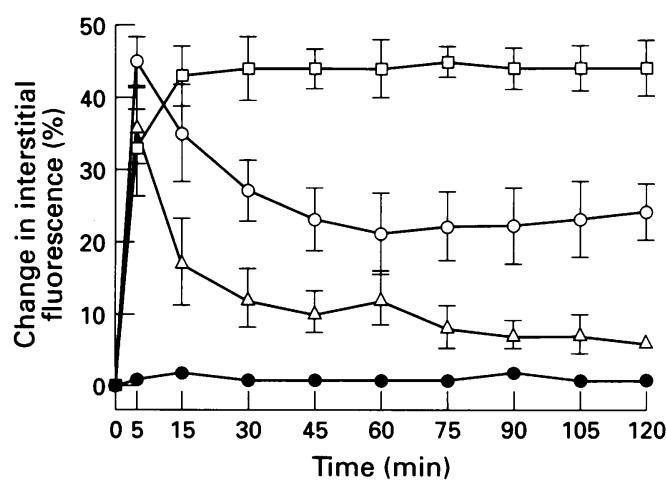

Figure 1: Effect of $60 \%$ ethanol on macromolecular leakage from superficial gastric mucosal postcapillary venules in lesion areas $\bigcirc$, areas peripheral to a lesion $\square$, areas remote from a lesion $\triangle$, and from control animals treated with distilled water $\bullet$. Results are means (SEM); $p<0.02$ for all values in lesion and peripheral sites and after five minutes in remote sites; $p<0.05$ for all other values in remote areas; $n=6$. 
PCVs. The same pattern of events was also found in the capillaries present in these areas.

LEUCOCYTE ADHERENCE AND ROLLING

In these six experimental animals only two randomly selected areas were observed and of these 12, six areas were at the periphery of blood flow stasis zones and the remainder were at a remote distance. There were no lesions in these preselected areas; therefore a separate examination was made throughout the course of the experiment to identify such areas and qualitatively assess leucocyte activity within them. Again, three distinctly separate responses to ethanol were identified according to the area.

In tissues often used for microcirculatory studies-for example, mesentery and cremaster muscle-rolling leucocytes are seen under control conditions. However, this is not seen in the gastric mucosal microcirculation (DN Granger; personal communication). Therefore, before the application of ethanol, no circulating leucocytes were seen in the microvessels. Adherent leucocytes were not seen either. After treatment, in the areas peripheral to blood flow stasis, a significant increase $(p<0.01)$ in the number of adherent leucocytes was seen after 30 minutes. Rolling leucocytes could now be seen circulating with a decreased velocity after treatment (Fig 2A). This effect was not as striking as in the remote zones where a signifiant increase $(p<0.01)$ in rolling leucocytes was observed after 15 minutes (Fig 2B).
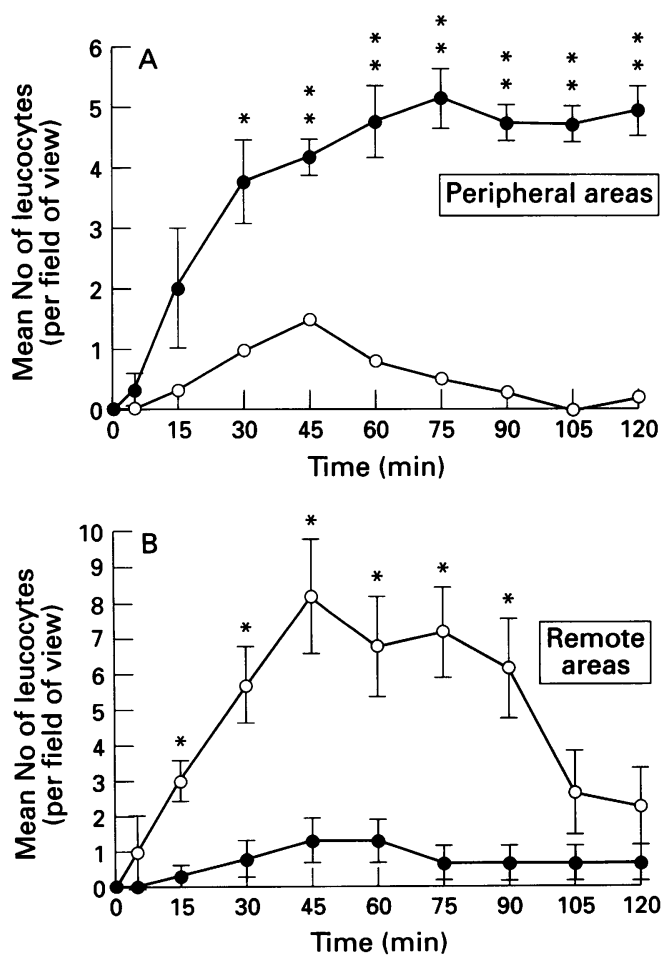

Figure 2: $(A)$ Effect of $60 \%$ ethanol on numbers of adherent $\bullet$ and rolling $\bigcirc$ leucocytes in superficial gastric mucosal vessels in areas peripheral to a lesion and $(B)$ areas remote from a lesion. Results are means (SEM); ${ }^{\star} p<0 \cdot 01$; ${ }^{\star *} p<0 \cdot 001 ; n=6$
In regions of blood flow stasis it was not possible to quantify adherent leucocytes, if any, due to the overwhelming numbers of congested red blood cells. Rolling leucocytes were not seen due to the lack of blood flow in these regions. Distilled water had no effect on the adherence or the velocity of circulating leucocytes. This provides good evidence of sustained tissue viability over the course of the experiment.

\section{Discussion}

Our findings suggest a close association between vascular stasis and mucosal necrosis and are consistent with previous studies in which different techniques have been employed. ${ }^{2-4}$ By simultaneously observing several areas of the mucosa we have been able to identify regional variations in vascular response, a feature not described so far. Of particular interest was that, despite uniform application of ethanol to the entire mucosa, widespread mucosal necrosis was not seen. The microcirculation was extensively perturbed as reflected by FITC-BSA leakage from all vessels, yet permanent damage, which is associated with irreversible stasis, was limited to small areas only. A particularly striking feature was the ability of the gastric microcirculation to recover despite extensive damage. We think that these changes are confined to the superficial microvasculature and that focal irreversible stasis occurs independently of vasospasm and leucocyte involvement. Presumably mechanisms exist (perhaps influence by local neuroendocrine factors), which produce changes to rapidly disperse the effects of ethanol and so minimise permanent damage.

As our findings are new we considered alternative mechanisms that might explain them. Theoretically, an artefact, caused by uneven contact between the ethanol and the mucosa, could be responsible. Unevenness in thickness of the mucus and variable exposure of the mucosa to ethanol caused by the natural folds in the stomach could result in lesions concentrated in areas of maximal contact. However, this is unlikely as when the stomach was opened the folds flattened, and before ethanol application, the mucus was carefully removed by absorption. Further evidence is provided by our parallel histological observations (not reported here) that showed superficial epithelial damage in areas where lesions did and did not form, indicating uniform mucosal exposure to ethanol. These findings have also been reported by others. ${ }^{9} 10$

We postulate that the focal necrosis was a result of changes in the superficial microcirculation independent of changes in vessel diameter. We did no find deeper changes in parallel histological studies (unpublished data). Nevertheless, mucosal necrosis could result from changes deeper down caused by submucosal venular constriction and associated arteriolar dilatation as has been suggested by Oates and Hakkinen. ${ }^{11}$ Their experimental system, however, was different 
from ours. They maintained overdistension of the pylorus ligated stomach of rats with $3 \mathrm{ml}$ ethanol, the aim being to specifically ensure that the damaging agent diffused to the submucosal vessels viewed from the external surface. By contrast, we applied only $0.5 \mathrm{ml}$ ethanol for five minutes.

Our findings could also be explained by the opening of arteriovenous shunts so limiting areas of damage, but such shunts, although searched for, have not been found. ${ }^{12}$

The mechanism of macromolecular leakage and the role of leucocytes require further comment. The leakage may result from a direct cytotoxic effect of ethanol on endothelial cells themselves or from release of histamine and $5 \mathrm{HT}$ via mucosal mast cell degranulation. ${ }^{13}$ Electron microscopy of the tissue five minutes after ethanol treatment showed the presence of gaps between the endothelial cells of vessels in all regions of the mucosa and also confirmed mast cell degranulation (unpublished data).

Leucocytes could theoretically contribute to ethanol induced gastric mucosal injury in several ways; oxygen derived free radical production, adherence to endothelium, and consequent release of vasoactive substances (leukotrienes, platelet activating factor) and by occlusion of the microcirculation. In our experiments, leucocytes adhered (and platelet thrombi formed) around the lesion, after their appearance and so presumably are not causal. However, we noted a close temporal association between adherence and sustained macromolecular leakage at the periphery of the lesions. Thus, leucocytes may not cause the lesions but may be important in sustaining leakage from the damaged microcirculation and perhaps exacerbating the injury. However, the role of the leucocytes already trapped within the sites of blood flow stasis is uncertain. Of particular interest is the finding of significant adherent leucocytes only around the lesion and rolling leucocytes only in remote areas. As selectins are involved in leucocyte rolling, it suggests that there is a rapid expression of selectins remotely with additional leucocyte activating signals for adherence near the lesion.
In summary, this study has shown that an intact microcirculation is essential in maintaining gastric mucosal integrity. In the face of uniform and widespread exposure of the mucosa to $60 \%$ ethanol, the resultant mucosal necrosis was minimal despite widespread vascular damage reflected by leakage of macromolecules, the pattern of which showed wide regional differences. Our findings raise the possibility that intrinsic protective mechanisms exist within the superficial gastric microcirculation which minimise mucosal damage.

$\mathrm{N} \mathrm{K}$ and this study were supported by the Bardhan Research and Education Trust; registered charity number 328452 .

1 Wodzinski MA, Bardhan KD, Reilly TJ, Cooper P, Preston FE. Reduced tissue type plasminogen activator of the gastroduodenal mucosa in peptic ulcer disease. Gut 1993; 34: 1310-14.

2 Pihan G, Majzoubi D, Haudenschild C, Trier JS, Szabo S. Early microcirculatory stasis in acute gastric mucosal injury in the rat and prevention by 16,16-dimethyl prostaglandin $\mathrm{E}_{2}$ or sodium thiosulfate. Gastroenterology prostaglandin $\mathrm{E}_{2}$ or

3 Bou-Abboud CF, Wayland H, Paulsen G, Guth PH. Microcirculatory stasis precedes tissue necrosis in
ethanol-induced gastric mucosal injury in the rat. Dig Dis Sci 1988; 33: 872-7.

4 Szabo S, Trier JS, Brown A, Schnoor J. Early vascular injury and increased vascular permeability in gastric mucosal injury caused by ethanol in the rat. Gastroenterology 1985; 88: 228-36.

5 Anthony A, Dhillon AP, Thrasivoulou, Pounder RE, Wakefield AJ. Occlusive intestinal vasculopathy by indomethacin: demonstration of carbon ink perfusion. $\mathcal{f}$ Pathol 1996 (suppl 1); 19A.

6 Piasecki C. Blood flow and ulceration: localizing mechanisms and ischaemic pathogenesis. In: Halter F, Garner A, Tytgat GNJ, eds. Mechanisms of peptic ulcer Garner A, Tytgat GNJ, eds. Mechanisms of peptic ulcer

7 Miller FN, Joshua IG, Anderson GL. Quantification of vasodilator-induced macromolecular leakage by in vivo fluorescent microscopy. Microvasc Res 1982; 24: 56-67.

8 Guth PH, Rosenburg A. In vivo microscopy of the gastric microcirculation. Dig Dis 1972; 17: 391-8.

9 Morris GP, Wallace JL. The roles of ethanol and of acid in the production of gastric mucosal erosions in rats. Virchows Archives 1981; 38: 23-38.

10 Lacy ER, Susumu I. Microscopic analysis of ethanol damage to the rat gastric mucosa after treatment with a prostaglandin. Gastroenterology 1982; 83: 619-25.

11 Oates PJ, Hakkinen P. Studies on the mechanism of ethanolinduced gastric damage in rats. Gastroenterology 1988; 94: induced $10-21$.

12 Gannon B, Browning J, O'Brien P. The microvascular architecture of the mucosa of the rat stomach. $\mathcal{F}$ Anat 1982; 135: 667-83.

13 Beck PL, Morris GP, Wallace JL. Reduction of ethanolinduced gastric damage by sodium cromoglycate and FPL-52694. Role of leukotrienes, prostaglandins and mast cells in the protective mechanism. Can $\mathcal{f}$ Physiol Pharmacol 1988; 67: 287-93. 